Вестник ЧГПУ им. И.Я. Яковлева

Серия: Механика предельного состояния. 2021. № 2. С. 48-62

\author{
А. Д. Чернышов ${ }^{1}$, В. В. Горяйнов ${ }^{2}$, С. Ф. Кузнецов ${ }^{1}$, О. Ю. Никифорова ${ }^{1}$ \\ ТРЕХМЕРНЫЕ ТОЧНЫЕ РЕШЕНИЯ УРАВНЕНИЯ ДИФФУЗИИ \\ ${ }^{1}$ Воронежский государственный университет инженерных технологий, г. Воронеж, \\ Россия, \\ ${ }^{2}$ Воронежский государственный технический университет, г. Воронеж, Россия
}

Аннотация. При помощи метода быстрых разложений решается задача диффузии в параллелепипеде с граничными условиями 1-го рода и внутренним источником вещества, зависящим от координат точек параллелепипеда. Получено в общем виде решение, содержащее свободные параметры, с помощью которых можно получить множество новых точных решений с различными свойствами. Показан пример построения точного решения для случая внутреннего источника переменного только по оси $O Z$. Приведен анализ особенностей диффузионных потоков в параллелепипеде с указанным внутреннем источником. Получено, что концентрация вещества в центре параллелепипеда равна сумме среднеарифметического значения концентраций вещества в его вершинах и амплитуды внутреннего источника умноженного на величину $c^{2} / \pi^{2}$.

Ключевые слова: аналитическое решение, точное решение, диффузионные потоки, переменный внутренний источник, параллелепипед, быстрые разложения.

DOI: 10.37972/chgpu.2021.48.2.007

УДК: 519.632

Введение. Для решения пространственных задач диффузии применяются различные численные и аналитические методы. В [1] на примере решения уравнения диффузии рассмотрен метод клеточных автоматов, как метод решения дифференциальных уравнений в частных производных параболического типа. В [2] рассмотрены

(C) Чернышов А. Д., Горяйнов В. В., Кузнецов С. Ф., Никифорова О. Ю., 2021

Чернышов Александр Данилович

e-mail: chernyshovad@mail.ru, д-р физ.-мат. наук, профессор, Воронежский государственный университет инженерных технологий, г. Воронеж, Россия.

Горяйнов Виталий Валервевич

e-mail: gorvit77@mail.ru, кандидат физико-математических наук, доцент, Воронежский государственный технический университет , г. Воронеж, Россия

Кузнецов Сергей Федорович

e-mail: sfs134@mail.ru, кандидат физико-математических наук, доцент, Воронежский государственный университет инженерных технологий, г. Воронеж, Россия

Никифорова Ольга Юрьевна

e-mail: niki22@mail.ru, старший преподаватель, Воронежский государственный университет инженерных технологий, г. Воронеж, Россия

Поступила 10.03.2021 
вопросы повышения эффективности параллельного многосеточного алгоритма решения разностных эллиптических трехмерных уравнений, который представляет собой параллельную реализацию классического многосеточного метода Р.П. Федоренко для краевых задач первого, второго и третьего рода, включая вырожденную задачу Неймана. Алгоритм эффективен для решения анизотропных задач, типичных в приложениях при моделировании процессов диффузии, теплопроводности, динамики жидкости. В работе [3] развивается метод наименьших квадратов с Т-элементами для решения линейных краевых задач с уравнениями Лапласа и Пуассона. Авторы используют разрывные базисные функции высокого порядка аппроксимации из специальных функциональных пространств. В [4] методом преобразования Фурье решается краевая задача Дирихле для уравнения Пуассона в области, ограниченной двумя параллельными гиперплоскостями в $R^{n}$. Решение представлено в виде суммы интегралов, ядра которых найдены в конечном виде. В [5] решение задачи об изгибе упругодиффузионной консольно-закрепленной балки Бернулли-Эйлера с учетом конечной скорости распространения диффузионных потоков ищется с помощью функций Грина, для нахождения которых используется преобразование Лапласа и разложение в тригонометрические ряды Фурье. Также среди численных методов для решения уравнения Пуассона следует отметить метод коллокаций [6], метод квадратурных элементов [7], модифицированный кубический В-сплайн дифференциально-квадратурный метод [8] и метод, основанный на использовании вейвлетов Хаара [9].

В данной работе будут использованы быстрые разложения [10], с помощью которых можно получать не только новые приближенные аналитические решения задач [1113], но и новые точные [14]. Применение быстрых разложений [10] позволит получить в общем виде решение краевой задачи о диффузии в параллелепипеде с переменным внутренним источником, точно удовлетворяющее дифференциальному уравнению и граничным условиям. Решение задачи содержит много свободных коэффициентов, которыми можно аппроксимировать широкий круг инженерных задач. В статье будет показано построение точного решения для случая внутреннего источника переменного по оси $O Z$.

Постановка задачи. Рассмотрим задачу диффузии в параллелепипеде $\Omega=$ $=\{(x, y, z) \in R, 0 \leq x \leq a, 0 \leq y \leq b, 0 \leq z \leq c\}$. Для неизвестной концентрации $C(x, y, z)$ запишем уравнение диффузии в форме Пуассона, как дифференциальное уравнение второго порядка в частных производных по переменным $x, y, z$ с заданным внутренним источником вещества $F(x, y, z)$ :

$$
\frac{\partial^{2} C}{\partial x^{2}}+\frac{\partial^{2} C}{\partial y^{2}}+\frac{\partial^{2} C}{\partial z^{2}}+F(x, y, z)=0
$$

Граничные условия на каждой грани параллелепипеда имеют вид

$$
\begin{aligned}
& \left.C\right|_{x=0}=f_{1}(y, z),\left.\quad C\right|_{y=0}=f_{2}(x, z),\left.\quad C\right|_{z=0}=f_{3}(x, y) \\
& \left.C\right|_{x=a}=f_{4}(y, z),\left.\quad C\right|_{y=b}=f_{5}(x, z),\left.\quad C\right|_{z=c}=f_{6}(x, y)
\end{aligned}
$$

Функции $f_{1} \div f_{6}$ в $(2)$ должны удовлетворять условиям совместности, так как концентрация $C(x, y, z)$, например, при подходе к ребру $(x=0, z=0)$ по двум смежным граням должна принимать одинаковые значения. Выполняя подобные условия на всех ребрах параллелепипеда, будем иметь 12 функциональных равенств 


$$
\begin{aligned}
& f_{1}(0, z)=f_{2}(0, z), \quad f_{1}(b, z)=f_{5}(0, z), \quad f_{2}(a, z)=f_{4}(0, z), \\
& f_{4}(b, z)=f_{5}(a, z), \quad f_{2}(x, 0)=f_{3}(x, 0) \quad f_{2}(x, c)=f_{6}(x, 0), \\
& f_{5}(x, 0)=f_{3}(x, b), \quad f_{5}(x, c)=f_{6}(x, b), \quad f_{1}(y, 0)=f_{3}(0, y) \\
& f_{1}(y, c)=f_{6}(0, y), \quad f_{4}(y, 0)=f_{3}(a, y), \quad f_{4}(y, c)=f_{6}(a, y) .
\end{aligned}
$$

Из условий независимости концентрации $C(x, y, z)$ от направления подхода к вершинам параллелепипеда получим следующие равенства

$$
\begin{aligned}
& f_{1}(0,0)=f_{2}(0,0)=f_{3}(0,0), \quad f_{1}(b, 0)=f_{3}(0, b)=f_{5}(0,0), \\
& f_{1}(0, c)=f_{2}(0, c)=f_{6}(0,0), \quad f_{2}(a, 0)=f_{3}(a, 0)=f_{4}(0,0), \\
& f_{2}(a, c)=f_{4}(0, c)=f_{6}(a, 0), \quad f_{1}(b, c)=f_{5}(0, c)=f_{6}(0, b), \\
& f_{3}(a, b)=f_{4}(b, 0)=f_{5}(a, 0), \quad f_{4}(b, c)=f_{5}(a, c)=f_{6}(a, b) .
\end{aligned}
$$

Равенства (3) и (4) позволяют записать условия выполнения дифференциального уравнения (1) в вершинах параллелепипеда:

$$
\begin{aligned}
& C_{x x}(0,0,0)+C_{y y}(0,0,0)+C_{z z}(0,0,0)+F(0,0,0)=0, \\
& C_{x x}(a, 0,0)+C_{y y}(a, 0,0)+C_{z z}(a, 0,0)+F(a, 0,0)=0, \\
& C_{x x}(0, b, 0)+C_{y y}(0, b, 0)+C_{z z}(0, b, 0)+F(0, b, 0)=0, \\
& C_{x x}(0,0, c)+C_{y y}(0,0, c)+C_{z z}(0,0, c)+F(0,0, c)=0, \\
& C_{x x}(a, b, 0)+C_{y y}(a, b, 0)+C_{z z}(a, b, 0)+F(a, b, 0)=0, \\
& C_{x x}(a, 0, c)+C_{y y}(a, 0, c)+C_{z z}(a, 0, c)+F(a, 0, c)=0, \\
& C_{x x}(0, b, c)+C_{y y}(0, b, c)+C_{z z}(0, b, c)+F(0, b, c)=0, \\
& C_{x x}(a, b, c)+C_{y y}(a, b, c)+C_{z z}(a, b, c)+F(a, b, c)=0 .
\end{aligned}
$$

Если же условия (3) и (4) не выполнять, то концентрация $C(x, y, z)$ будет терпеть разрыв на ребрах и в вершинах параллелепипеда, что физически противоречиво. Если же не выполнять равенства (5), то будет нарушаться закон сохранения массы в вершинах параллелепипеда.

При построении решения задачи о распределении концентрации вещества $C(x, y, z)$ можно использовать часть полиномов и тригонометрических функций, которые возникают в теории быстрых разложений. Этих функций будет достаточно, чтобы точно удовлетворить и дифференциальному уравнению (1), граничным условиям (2) и условиям согласований (3)-(5). Предполагаемое точное решение запишем следующим конечным выражением

$$
\begin{gathered}
C(x, y, z)=\sum_{i=1}^{2} A_{i}(y, z) P_{i}(x)+A_{3}(y, z) \sin \frac{\pi x}{a}+A_{4}(y, z) \sin \frac{2 \pi x}{a}, 0 \leq x \leq a \\
A_{i}(y, z)=\sum_{j=1}^{2} A_{i, j}(z) P_{j}(y)+A_{i, 3}(z) \sin \frac{\pi y}{b}+A_{i, 4}(z) \sin \frac{2 \pi y}{b} \\
i=1 \div 4,0 \leq y \leq b, \\
A_{i, j}(z)=\sum_{k=1}^{2} A_{i, j, k} P_{k}(z)+A_{i, j, 3} \sin \frac{\pi z}{c}+A_{i, j, 4} \sin \frac{2 \pi z}{c}, \quad j=1 \div 4,0 \leq z \leq c \\
P_{1}(x)=1-\frac{x}{a}, \quad P_{2}(x)=\frac{x}{a}, P_{1}(y)=1-\frac{y}{b}, \quad P_{2}(y)=\frac{y}{b} \\
P_{1}(z)=1-\frac{z}{c}, P_{2}(z)=\frac{z}{c} .
\end{gathered}
$$


Для удобства дальнейших преобразований введем обозначения:

$$
\begin{aligned}
& \sin \frac{\pi x}{a}=P_{3}(x), \quad \sin \frac{2 \pi x}{a}=P_{4}(x), \sin \frac{\pi y}{b}=P_{3}(y), \\
& \sin \frac{2 \pi y}{b}=P_{4}(y), \sin \frac{\pi z}{c}=P_{3}(z), \sin \frac{2 \pi z}{c}=P_{4}(z) .
\end{aligned}
$$

Тогда зависимость $C(x, y, z)$ из (6) можно представить более кратко

$$
\begin{gathered}
C(x, y, z)=\sum_{i=1}^{4}\left(\sum_{j=1}^{4}\left(\sum_{k=1}^{4} A_{i, j, k} P_{k}(z)\right) P_{j}(y)\right) P_{i}(x) \\
0 \leq x \leq a, \quad 0 \leq y \leq b, 0 \leq z \leq c .
\end{gathered}
$$

Таким образом, искомая функция $C(x, y, z)$ представлена в виде конечной тройной суммы, содержащей 64 неизвестных коэффициента

$$
A_{i, j, k}, \quad i=1 \div 4, \quad j=1 \div 4, \quad k=1 \div 4 .
$$

Для получения точного решения внутренний источник вещества $F(x, y, z)$ представим конечной суммой по аналогии с зависимостью (7):

$$
\begin{aligned}
F(x, y, z) & =\sum_{i=1}^{4}\left(\sum_{j=1}^{4}\left(\sum_{k=1}^{4} F_{i, j, k} P_{k}(z)\right) P_{j}(y)\right) P_{i}(x), \\
0 & \leq x \leq a, \quad 0 \leq y \leq b, 0 \leq z \leq c .
\end{aligned}
$$

Все коэффициенты $F_{i, j, k}$ в (9) для источника считаем известными.

В граничных условиях (2) функции $f_{1}(y, z), f_{2}(x, z), f_{3}(x, y), f_{4}(y, z)$, $f_{5}(x, z), \quad f_{6}(x, y)$ зададим следующим образом

$$
\begin{aligned}
& f_{1}(y, z)=\sum_{j=1}^{4}\left(\sum_{k=1}^{4} f_{1, j, k} P_{k}(z)\right) P_{j}(y) \\
& f_{2}(x, z)=\sum_{j=1}^{4}\left(\sum_{k=1}^{4} f_{2, j, k} P_{k}(z)\right) P_{j}(x) \\
& f_{3}(x, y)=\sum_{j=1}^{4}\left(\sum_{k=1}^{4} f_{3, j, k} P_{k}(y)\right) P_{j}(x) \\
& f_{4}(y, z)=\sum_{j=1}^{4}\left(\sum_{k=1}^{4} f_{4, j, k} P_{k}(z)\right) P_{j}(y) \\
& f_{5}(x, z)=\sum_{j=1}^{4}\left(\sum_{k=1}^{4} f_{5, j, k} P_{k}(z)\right) P_{j}(x) \\
& f_{6}(x, y)=\sum_{j=1}^{4}\left(\sum_{k=1}^{4} f_{6, j, k} P_{k}(y)\right) P_{j}(x) .
\end{aligned}
$$

Здесь $f_{i, j, k}$ считаем известными постоянными.

Приходим к следующей задаче: требуется найти гладкое точное решение уравнения (1) с заданным внутренним источником (9), удовлетворяющее граничным условиям (2) и условиям согласований (3)-(5).

Коэффициенты $A_{i, j, k}$ из (8), которые определяют решение задачи, считаем пока неизвестными. Их надо найти при помощи точного выполнения граничных условий (2), условий согласований (3)-(5) и дифференциального уравнения (1). 
Решение задачи. Предполагаемую зависимость (7) подставим в условия согласований (3), (4) и (5):

$$
\begin{gathered}
f_{1,1,1}=f_{2,1,1}=f_{3,1,1}, \quad f_{1,1,2}=f_{2,1,2}=f_{6,1,1}, \\
f_{1,2,1}=f_{5,1,1}=f_{3,1,2}, \quad f_{1,2,2}=f_{5,1,2}=f_{6,1,2}, \\
f_{2,2,1}=f_{4,1,1}=f_{3,2,1}, \quad f_{2,2,2}=f_{4,1,2}=f_{6,2,1}, \\
f_{4,2,1}=f_{5,2,1}=f_{3,2,2}, \quad f_{4,2,2}=f_{5,2,2}=f_{6,2,2}, \\
f_{1,1,3}=f_{2,1,3}, \quad f_{1,1,4}=f_{2,1,4}, f_{1,2,3}=f_{5,1,3}, \quad f_{1,2,4}=f_{5,1,4}, \\
f_{1,3,1}=f_{3,1,3}, \quad f_{1,3,2}=f_{6,1,3}, f_{1,4,1}=f_{3,1,4}, \quad f_{1,4,2}=f_{6,1,4}, \\
f_{2,2,3}=f_{4,1,3}, \quad f_{2,2,4}=f_{4,1,4}, f_{4,2,3}=f_{5,2,3}, \quad f_{4,2,4}=f_{5,2,4}, \\
f_{4,3,1}=f_{3,2,3}, \quad f_{4,3,2}=f_{6,2,3}, f_{4,4,1}=f_{3,2,4}, \quad f_{4,4,2}=f_{6,2,4}, \\
f_{2,3,1}=f_{3,3,1}, \quad f_{2,3,2}=f_{6,3,1}, f_{5,3,1}=f_{3,3,2}, \quad f_{5,3,2}=f_{6,3,2}, \\
f_{2,4,1}=f_{3,4,1}, \quad f_{2,4,2}=f_{6,4,1}, f_{5,4,1}=f_{3,4,2}, \quad f_{5,4,2}=f_{6,4,2} . \\
F_{1,1,1}=F_{1,1,2}=F_{1,2,1}=F_{1,2,2}=F_{2,1,1}=F_{2,1,2}=F_{2,2,1}=F_{2,2,2}=0 .
\end{gathered}
$$

После подстановки предполагаемого решения задачи в форме (7) в граничные условия (2) и дифференциальное уравнение (1), получим функциональную систему, которую сведем к системе линейных алгебраических уравнений путем приравнивания коэффициентов слева и справа перед линейно независимыми функциями

$$
P_{1}(x) \div P_{4}(x), P_{1}(y) \div P_{4}(y), P_{1}(z) \div P_{4}(z) \text {. }
$$

Полученная система будет состоять из 160 линейных алгебраических уравнений, которую обозначим через $S$. Система $S$ будет переопределенной. Для нахождения неизвестных $A_{i, j, k}$ из (8) необходимо выполнить 64 независимых алгебраических уравнений из $S$. Остальные 96 уравнений являются линейно зависимыми, 48 из которых автоматически удовлетворяют условиям согласований (3)-(5), оставшиеся 48 уравнений используем для составления связи между коэффициентами $f_{i, j, k}$ и $F_{i, j, k}$, $i=1 \div 4, \quad j=1 \div 4, \quad k=1 \div 4$.

В результате имеем следующие значения коэффициентов $A_{i, j, k}$, определяющие точное решение:

$$
\begin{aligned}
& A_{1, j, k}=f_{1, j, k}, \quad j=1 \div 4, \quad k=1 \div 4 \\
& A_{2,1, k}=f_{2,2, k}, \quad k=1 \div 4 ; \\
& A_{2, j, k}=f_{4, j, k}, \quad j=2,3,4, \quad k=1 \div 4 \\
& A_{3,1, k}=f_{2,3, k}, \quad A_{3,2, k}=f_{5,3, k}, \quad k=1 \div 4 \\
& A_{3,3,1}=f_{3,3,3}, \quad A_{3,3,2}=f_{6,3,3}, A_{3,3,3}=F_{3,3,3} /\left(\frac{\pi^{2}}{a^{2}}+\frac{\pi^{2}}{b^{2}}+\frac{\pi^{2}}{c^{2}}\right), \\
& A_{3,3,4}=F_{3,3,4} /\left(\frac{\pi^{2}}{a^{2}}+\frac{\pi^{2}}{b^{2}}+\frac{4 \pi^{2}}{c^{2}}\right), \\
& A_{3,4,1}=f_{3,3,4}, \quad A_{3,4,2}=f_{6,3,4}, \\
& A_{3,4,3}=F_{3,4,3} /\left(\frac{\pi^{2}}{a^{2}}+\frac{4 \pi^{2}}{b^{2}}+\frac{\pi^{2}}{c^{2}}\right), \\
& A_{3,4,4}=F_{3,4,4} /\left(\frac{\pi^{2}}{a^{2}}+\frac{4 \pi^{2}}{b^{2}}+\frac{4 \pi^{2}}{c^{2}}\right),
\end{aligned}
$$




$$
\begin{aligned}
& A_{4,1, k}=f_{2,4, k}, \quad A_{4,2, k}=f_{5,4, k}, \quad k=1 \div 4 ; \\
& A_{4,3,1}=f_{3,4,3}, \quad A_{4,3,2}=f_{6,4,3}, \\
& A_{4,3,3}=F_{4,3,3} /\left(\frac{4 \pi^{2}}{a^{2}}+\frac{\pi^{2}}{b^{2}}+\frac{\pi^{2}}{c^{2}}\right), \\
& A_{4,3,4}=F_{4,3,4} /\left(\frac{4 \pi^{2}}{a^{2}}+\frac{\pi^{2}}{b^{2}}+\frac{4 \pi^{2}}{c^{2}}\right), \\
& A_{4,4,1}=f_{3,4,4}, \quad A_{4,4,2}=f_{6,4,4}, \\
& A_{4,4,3}=F_{4,4,3} /\left(\frac{4 \pi^{2}}{a^{2}}+\frac{4 \pi^{2}}{b^{2}}+\frac{\pi^{2}}{c^{2}}\right), \\
& A_{4,4,4}=F_{4,4,4} /\left(\frac{4 \pi^{2}}{a^{2}}+\frac{4 \pi^{2}}{b^{2}}+\frac{4 \pi^{2}}{c^{2}}\right) .
\end{aligned}
$$

Подставив коэффициенты (14) в выражение (7), будем иметь аналитическое решение задачи. Данное решение имеет место, когда выполнены алгебраические соотношение (11)-(13) и дополнительные равенства:

$$
\begin{aligned}
& f_{1, j, 3}=\frac{c^{2}}{\pi^{2}} F_{1, j, 3}, j=1,2 ; f_{1, j, 4}=\frac{c^{2}}{4 \pi^{2}} F_{1, j, 4}, j=1,2 \\
& f_{1,3, k}=\frac{b^{2}}{\pi^{2}} F_{1,3, k}, \quad k=1,2 ; f_{1,3,3}=F_{1,3,3} /\left(\frac{\pi^{2}}{b^{2}}+\frac{\pi^{2}}{c^{2}}\right) \\
& f_{1,3,4}=F_{1,3,4} /\left(\frac{\pi^{2}}{b^{2}}+\frac{4 \pi^{2}}{c^{2}}\right), f_{1,4, k}=\frac{b^{2}}{4 \pi^{2}} F_{1,4, k}, \quad k=1,2 \\
& f_{1,4,3}=F_{1,4,3} /\left(\frac{4 \pi^{2}}{b^{2}}+\frac{\pi^{2}}{c^{2}}\right), f_{1,4,4}=F_{1,4,4} /\left(\frac{4 \pi^{2}}{b^{2}}+\frac{4 \pi^{2}}{c^{2}}\right), \\
& f_{2,2,3}=\frac{c^{2}}{\pi^{2}} F_{2,1,3}, \quad f_{2,2,4}=\frac{c^{2}}{4 \pi^{2}} F_{2,1,4}, f_{4,2,3}=\frac{c^{2}}{\pi^{2}} F_{2,2,3}, f_{4,2,4}=\frac{c^{2}}{4 \pi^{2}} F_{2,2,4}, \\
& f_{4,3, k}=\frac{b^{2}}{\pi^{2}} F_{2,3, k}, \quad k=1,2 ; f_{4,3,3}=F_{2,3,3} /\left(\frac{\pi^{2}}{b^{2}}+\frac{\pi^{2}}{c^{2}}\right) \\
& f_{4,3,4}=F_{2,3,4} /\left(\frac{\pi^{2}}{b^{2}}+\frac{4 \pi^{2}}{c^{2}}\right), f_{4,4, k}=\frac{b^{2}}{4 \pi^{2}} F_{2,4, k}, k=1,2 \\
& f_{4,4,3}=F_{2,4,3} /\left(\frac{4 \pi^{2}}{b^{2}}+\frac{\pi^{2}}{c^{2}}\right), f_{4,4,4}=F_{2,4,4} /\left(\frac{4 \pi^{2}}{b^{2}}+\frac{4 \pi^{2}}{c^{2}}\right) \\
& f_{2,3, k}=\frac{a^{2}}{\pi^{2}} F_{3,1, k}, k=1,2 ; f_{2,3,3}=F_{3,1,3} /\left(\frac{\pi^{2}}{a^{2}}+\frac{\pi^{2}}{c^{2}}\right) \\
& f_{2,3,4}=F_{3,1,4} /\left(\frac{\pi^{2}}{a^{2}}+\frac{4 \pi^{2}}{c^{2}}\right), f_{5,3, k}=\frac{a^{2}}{\pi^{2}} F_{3,2, k}, k=1,2 \\
& f_{5,3,3}=F_{3,2,3} /\left(\frac{\pi^{2}}{a^{2}}+\frac{\pi^{2}}{c^{2}}\right), f_{5,3,4}=F_{3,2,4} /\left(\frac{\pi^{2}}{a^{2}}+\frac{4 \pi^{2}}{c^{2}}\right) \\
& f_{3,3,3}=F_{3,3,1} /\left(\frac{\pi^{2}}{a^{2}}+\frac{\pi^{2}}{b^{2}}\right), f_{6,3,3}=F_{3,3,2} /\left(\frac{\pi^{2}}{a^{2}}+\frac{\pi^{2}}{b^{2}}\right) \\
& f_{3,3,4}=F_{3,4,1} /\left(\frac{\pi^{2}}{a^{2}}+\frac{4 \pi^{2}}{b^{2}}\right), f_{6,3,4}=F_{3,4,2} /\left(\frac{\pi^{2}}{a^{2}}+\frac{4 \pi^{2}}{b^{2}}\right) \\
& f_{2,4, k}=\frac{a^{2}}{4 \pi^{2}} F_{4,1, k}, k=1,2 ; f_{2,4,3}=F_{4,1,3} /\left(\frac{4 \pi^{2}}{a^{2}}+\frac{\pi^{2}}{c^{2}}\right) \\
& \left.f^{2}\right)
\end{aligned}
$$




$$
\begin{aligned}
& f_{2,4,4}=F_{4,1,4} /\left(\frac{4 \pi^{2}}{a^{2}}+\frac{4 \pi^{2}}{c^{2}}\right), f_{5,4, k}=\frac{a^{2}}{4 \pi^{2}} F_{4,2, k}, k=1,2 ; \\
& f_{5,4,3}=F_{4,2,3} /\left(\frac{4 \pi^{2}}{a^{2}}+\frac{\pi^{2}}{c^{2}}\right), f_{5,4,4}=F_{4,2,4} /\left(\frac{4 \pi^{2}}{a^{2}}+\frac{4 \pi^{2}}{c^{2}}\right), \\
& f_{3,4,3}=F_{4,3,1} /\left(\frac{4 \pi^{2}}{a^{2}}+\frac{\pi^{2}}{b^{2}}\right), f_{6,4,3}=F_{4,3,2} /\left(\frac{4 \pi^{2}}{a^{2}}+\frac{\pi^{2}}{b^{2}}\right), \\
& f_{3,4,4}=F_{4,4,1} /\left(\frac{4 \pi^{2}}{a^{2}}+\frac{4 \pi^{2}}{b^{2}}\right), f_{6,4,4}=F_{4,4,2} /\left(\frac{4 \pi^{2}}{a^{2}}+\frac{4 \pi^{2}}{b^{2}}\right) .
\end{aligned}
$$

Пример построения точного решения и его анализ. Зададим внутренний источник, действующий в параллелепипеде, следующим образом. Примем в (9) $F_{3}(y, z)=F_{4}(y, z)=0$, тогда источник будет иметь вид

$$
F(x, y, z)=F_{1}(y, z) P_{1}(x)+F_{2}(y, z) P_{2}(x) .
$$

В функциях $F_{1}(y, z)$ и $F_{2}(y, z)$ из $(16)$ положим $F_{1,3}(z)=F_{1,4}(z)=F_{2,3}(z)=$ $F_{2,4}(z)=0$. В результате получим

$$
\begin{aligned}
& F_{1}(y, z)=F_{1,1}(z) P_{1}(y)+F_{1,2}(z) P_{2}(y) \\
& F_{2}(y, z)=F_{2,1}(z) P_{1}(y)+F_{2,2}(z) P_{2}(y) .
\end{aligned}
$$

В представлении функций $F_{1,1}(z), F_{1,2}(z), F_{2,1}(z), F_{2,2}(z)$ зададим $F_{1,1,4}=F_{1,2,4}=$ $F_{2,1,4}=F_{2,2,4}=0$ и, учитывая равенства (13), указанные функции примут вид

$$
\begin{aligned}
& F_{1,1}(z)=F_{1,1,3} \sin \pi \frac{z}{c}, F_{1,2}(z)=F_{1,2,3} \sin \pi \frac{z}{c}, \\
& F_{2,1}(z)=F_{2,1,3} \sin \pi \frac{z}{c}, F_{2,2}(z)=F_{2,2,3} \sin \pi \frac{z}{c} .
\end{aligned}
$$

Используя обозначения в (6), окончательно запишем

$$
\begin{gathered}
F(x, y, z)=\left[F_{1,1,3}\left(1-\frac{y}{b}\right) \sin \pi \frac{z}{c}+F_{1,2,3} \frac{y}{b} \sin \pi \frac{z}{c}\right]\left(1-\frac{x}{a}\right)+ \\
+\left[F_{2,1,3}\left(1-\frac{y}{b}\right) \sin \pi \frac{z}{c}+F_{2,2,3} \frac{y}{b} \sin \pi \frac{z}{c}\right] \frac{x}{a} .
\end{gathered}
$$

При выполнении равенств $F_{1,1,3}=F_{1,2,3}=F_{2,1,3}=F_{2,2,3}=Q$ внутренний источник в (17) будет зависеть только от координаты $z$ и не будет зависеть от $x$ и $y$ :

$$
F(x, y, z)=Q \sin \pi \frac{z}{c} .
$$

Запишем граничные условия, которые будут выполняться для источника (18). Пусть в равенствах (10) некоторые функции равны нулю

$$
f_{1, j}(z)=f_{2, j}(z)=f_{3, j}(y)=f_{4, j}(z)=f_{5, j}(z)=f_{6, j}(y)=0, \quad j=3 ; 4,
$$

а также равны нулю коэффициенты

$$
\begin{gathered}
f_{1,1,4}=f_{1,2,4}=f_{2,1,4}=f_{2,2,4}=f_{3,1,4}=f_{3,2,4}= \\
f_{4,1,4}=f_{4,2,4}=f_{5,1,4}=f_{5,2,4}=f_{6,1,4}=f_{6,2,4}=0 .
\end{gathered}
$$


В этом случае граничные условия (2) с учетом равенств (11), (12), (15) и обозначений

примут вид

$$
\begin{aligned}
& f_{1,1,1}=f_{2,1,1}=f_{3,1,1}=C_{1}, f_{1,1,2}=f_{2,1,2}=f_{6,1,1}=C_{2}, \\
& f_{1,1,3}=f_{2,1,3}=\frac{c^{2}}{\pi^{2}} Q, f_{1,2,1}=f_{5,1,1}=f_{3,1,2}=C_{3}, \\
& f_{1,2,2}=f_{5,1,2}=f_{6,1,2}=C_{4}, f_{1,2,3}=f_{5,1,3}=\frac{c^{2}}{\pi^{2}} Q, \\
& f_{2,2,1}=f_{4,1,1}=f_{3,2,1}=C_{5}, f_{2,2,2}=f_{4,1,2}=f_{6,2,1}=C_{6}, \\
& f_{2,2,3}=f_{4,1,3}=\frac{c^{2}}{\pi^{2}} Q, f_{4,2,1}=f_{5,2,1}=f_{3,2,2}=C_{7}, \\
& f_{4,2,2}=f_{5,2,2}=f_{6,2,2}=C_{8}, f_{4,2,3}=f_{5,2,3}=\frac{c^{2}}{\pi^{2}} Q,
\end{aligned}
$$

$$
\begin{aligned}
& \left.C\right|_{x=0}=\left(C_{1}\left(1-\frac{z}{c}\right)+C_{2} \frac{z}{c}\right)\left(1-\frac{y}{b}\right)+\left(C_{3}\left(1-\frac{z}{c}\right)+C_{4} \frac{z}{c}\right) \frac{y}{b}+ \\
& +\frac{c^{2}}{\pi^{2}} Q \sin \pi \frac{z}{c}, \\
& \left.C\right|_{y=0}=\left(C_{1}\left(1-\frac{z}{c}\right)+C_{2} \frac{z}{c}\right)\left(1-\frac{x}{a}\right)+\left(C_{5}\left(1-\frac{z}{c}\right)+C_{6} \frac{z}{c}\right) \frac{x}{a}+ \\
& +\frac{c^{2}}{\pi^{2}} Q \sin \pi \frac{z}{c} \\
& \left.C\right|_{z=0}=\left(C_{1}\left(1-\frac{y}{b}\right)+C_{3} \frac{y}{b}\right)\left(1-\frac{x}{a}\right)+\left(C_{5}\left(1-\frac{y}{b}\right)+C_{7} \frac{y}{b}\right) \frac{x}{a} \\
& \left.C\right|_{x=a}=\left(C_{5}\left(1-\frac{z}{c}\right)+C_{6} \frac{z}{c}\right)\left(1-\frac{y}{b}\right)+\left(C_{7}\left(1-\frac{z}{c}\right)+C_{8} \frac{z}{c}\right) \frac{y}{b}+ \\
& +\frac{c^{2}}{\pi^{2}} Q \sin \pi \frac{z}{c}, \\
& \left.C\right|_{y=b}=\left(C_{3}\left(1-\frac{z}{c}\right)+C_{4} \frac{z}{c}\right)\left(1-\frac{x}{a}\right)+\left(C_{7}\left(1-\frac{z}{c}\right)+C_{8} \frac{z}{c}\right) \frac{x}{a}+ \\
& +\frac{c^{2}}{\pi^{2}} Q \sin \pi \frac{z}{c}, \\
& \left.C\right|_{z=c}=\left(C_{2}\left(1-\frac{y}{b}\right)+C_{4} \frac{y}{b}\right)\left(1-\frac{x}{a}\right)+\left(C_{6}\left(1-\frac{y}{b}\right)+C_{8} \frac{y}{b}\right) \frac{x}{a} .
\end{aligned}
$$

Подставляя в (14) коэффициенты из (19) и, учитывая принятые равными нулю коэффициенты из граничных условий и внутреннего источника, получим

$$
\begin{gathered}
A_{1,1,1}=C_{1}, A_{1,1,2}=C_{2}, A_{1,1,3}=\frac{c^{2}}{\pi^{2}} Q, A_{1,2,1}=C_{3}, A_{1,2,2}=C_{4}, \\
A_{1,2,3}=\frac{c^{2}}{\pi^{2}} Q, A_{2,1,1}=C_{5}, A_{2,1,2}=C_{6}, A_{2,1,3}=\frac{c^{2}}{\pi^{2}} Q, A_{2,2,1}=C_{7}, \\
A_{2,2,2}=C_{8}, A_{2,2,3}=\frac{c^{2}}{\pi^{2}} Q .
\end{gathered}
$$

После упрощений точное решение уравнения (1), соответствующее условиям (20), с внутренним источником (18) принимает вид

$$
\begin{aligned}
C(x, y, z)=[ & \left.\left(C_{1}\left(1-\frac{z}{c}\right)+C_{2} \frac{z}{c}\right)\left(1-\frac{y}{b}\right)+\left(C_{3}\left(1-\frac{z}{c}\right)+C_{4} \frac{z}{c}\right) \frac{y}{b}\right] \times \\
& \times\left(1-\frac{x}{a}\right)+\left[\left(C_{5}\left(1-\frac{z}{c}\right)+C_{6} \frac{z}{c}\right)\left(1-\frac{y}{b}\right)+\right. \\
& \left.+\left(C_{7}\left(1-\frac{z}{c}\right)+C_{8} \frac{z}{c}\right) \frac{y}{b}\right] \frac{x}{a}+\frac{c^{2}}{\pi^{2}} Q \sin \pi \frac{z}{c} .
\end{aligned}
$$

По формуле (21) можно вычислить концентрацию вещества $C(x, y, z)$ в любой точке параллелепипеда. В частности, подставляя в (21) $x=a / 2, y=b / 2$ и $z=c / 2$, вычислим 


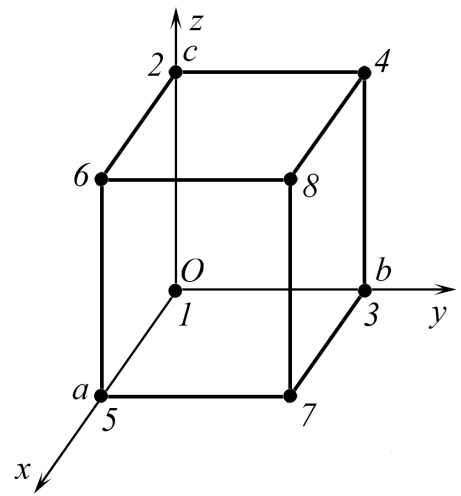

Рис. 1. Параллелепипед

значение концентрации в центре тела

$$
C\left(\frac{a}{2}, \frac{b}{2}, \frac{c}{2}\right)=\frac{c^{2}}{\pi^{2}} Q+\frac{C_{1}+C_{2}+C_{3}+C_{4}+C_{5}+C_{6}+C_{7}+C_{8}}{8}
$$

Из (22) следует, что концентрация вещества в центре параллелепипеда равна сумме среднеарифметического значения концентраций вещества в его вершинах (рис. 1) и амплитуды $Q$ внутреннего источника умноженного на величину $c^{2} / \pi^{2}$.

Вычислим диффузионные потоки вещества по формулам [15]

$$
\begin{aligned}
& q_{x}(x, y, z)=-D \frac{\partial C(x, y, z)}{\partial x}, q_{y}(x, y, z)=-D \frac{\partial C(x, y, z)}{\partial y}, \\
& q_{z}(x, y, z)=-D \frac{\partial C(x, y, z)}{\partial z} .
\end{aligned}
$$

где $D$ - коэффициент диффузии.

Подставляя в (23) точное решение (21), после преобразований будем иметь

$$
\begin{gathered}
q_{x}(x, y, z)=-\frac{D}{a}\left(-\left(C_{1}-C_{5}\right)+\frac{z}{c}\left(\left(C_{1}-C_{5}\right)-\left(C_{2}-C_{6}\right)\right)+\right. \\
+\frac{y}{b}\left(\left(C_{1}-C_{5}\right)-\left(C_{3}-C_{7}\right)\right)+ \\
\left.+\frac{z}{c} \frac{y}{b}\left(\left(C_{2}-C_{6}\right)-\left(C_{1}-C_{5}\right)+\left(C_{3}-C_{7}\right)-\left(C_{4}-C_{8}\right)\right)\right), \\
q_{y}(x, y, z)=-\frac{D}{b}\left(-\left(C_{1}-C_{3}\right)+\frac{z}{c}\left(\left(C_{1}-C_{3}\right)-\left(C_{2}-C_{4}\right)\right)+\right. \\
\left.\left.+\frac{z}{c} \frac{x}{a}\left(\left(C_{2}-C_{4}\right)-\left(C_{1}-C_{3}\right)+\left(C_{5}-C_{7}\right)\right)+\left(C_{6}-C_{8}\right)\right)\right), \\
q_{z}(x, y, z)=-\frac{D}{c}\left(\frac{c^{2}}{\pi} Q \cos \pi \frac{z}{c}-\left(C_{1}-C_{2}\right)+\frac{y}{b}\left(\left(C_{1}-C_{2}\right)-\left(C_{3}-C_{4}\right)\right)+\right. \\
+\frac{x}{b}\left(\left(C_{1}-C_{2}\right)-\left(C_{5}-C_{6}\right)\right)+ \\
\left.\frac{y}{a}\left(-\left(C_{1}-C_{2}\right)+\left(C_{3}-C_{4}\right)+\left(C_{5}-C_{6}\right)-\left(C_{7}-C_{8}\right)\right)\right) .
\end{gathered}
$$

Из (24)-(26) видно, что потоки $q_{x}(x, y, z)$ и $q_{y}(x, y, z)$ не зависят от амплитуды внутреннего источника $Q$, а зависят только от концентраций вещества в вершинах 
параллелепипеда (рис. 1). Величины потоков $q_{x}(x, y, z)$ и $q_{y}(x, y, z)$ обратно пропорциональны соответствующим геометрическим размерам параллелепипеда $a$ и $b$. На поток $q_{z}(x, y, z)$ влияют и концентрации вещества в вершинах, и амплитуда внутреннего источника, причем вклад слагаемого, содержащего внутренний источник, тем больше чем больше высота параллелепипеда $c$.

В формулах (24)-(26) концентрации $C_{i}, i=1 . .8$ сгруппированы так, чтобы выделить разность концентраций вещества в вершинах на данном ребре параллелепипеда (рис. 1). Таким образом, из (24)-(26) видно, что в описании потока $q_{x}(x, y, z)$ присутствуют разности концентраций в вершинах на ребрах коллинеарных только оси $O X$ (формула $(24))$. Для описания $q_{y}(x, y, z)$ учитываются разности концентраций в вершинах на ребрах коллинеарных только оси $O Y$ (формула (25)), а в формуле (26) в записи $q_{z}(x, y, z)$ присутствуют разности концентраций вещества в вершинах на ребрах коллинеарных только оси $O Z$.

Введем обозначения для разности концентраций в вершинах на соответствующих ребрах

$$
\begin{aligned}
& \Delta C_{2,6}=C_{2}-C_{6}, \Delta C_{1,5}=C_{1}-C_{5}, \quad \Delta C_{3,7}=C_{3}-C_{7}, \Delta C_{4,8}=C_{4}-C_{8}, \\
& \Delta C_{2,4}=C_{2}-C_{4}, \Delta C_{1,3}=C_{1}-C_{3}, \quad \Delta C_{5,7}=C_{5}-C_{7}, \Delta C_{6,8}=C_{6}-C_{8}, \\
& \Delta C_{3,4}=C_{3}-C_{4}, \Delta C_{1,2}=C_{1}-C_{2}, \Delta C_{5,6}=C_{5}-C_{6}, \Delta C_{7,8}=C_{7}-C_{8} .
\end{aligned}
$$

Описание потоков $q_{x}(x, y, z), q_{y}(x, y, z)$ и $q_{z}(x, y, z)$ формулами $(24)$-(26) показывает, что возможны случаи качественно различных профилей $q_{x}(x, y, z), q_{y}(x, y, z)$ и $q_{z}(x, y, z)$ в зависимости от того сколько будет одинаковых значений разности концентраций $\Delta C_{i, j}, i=1 \ldots 7, j=2 \ldots 8$ в формулах $(27)-(29)$.

Если в равенствах (27)-(29) все $\Delta C_{i, j}, i=1 \ldots 7, j=2 \ldots 8$ одинаковые, т.е.

$$
\begin{gathered}
\Delta C_{2,6}=\Delta C_{1,5}=\Delta C_{3,7}=\Delta C_{4,8}, \Delta C_{2,4}=\Delta C_{1,3}=\Delta C_{5,7}=\Delta C_{6,8}, \\
\Delta C_{3,4}=\Delta C_{1,2}=\Delta C_{5,6}=\Delta C_{7,8},
\end{gathered}
$$

то будем иметь следующие выражения для потоков вещества

$$
\begin{gathered}
q_{x}(x, y, z)=\frac{D}{a} \Delta C_{1,5}, q_{y}(x, y, z)=\frac{D}{b} \Delta C_{1,3}, \\
q_{z}(x, y, z)=\frac{D}{c} \Delta C_{1,2}-D \frac{c}{\pi} Q \cos \pi \frac{z}{c} .
\end{gathered}
$$

Из (31) следует, что при выполнении условий (30) потоки $q_{x}(x, y, z)$ и $q_{y}(x, y, z)$ будут постоянными, а $q_{z}(x, y, z)$ переменен только по оси $O Z$.

Если в равенствах (27)-(29) три значения из четырех $\Delta C_{i, j}, i=1 \ldots 7, j=2 \ldots 8$ одинаковые, например

$$
\begin{aligned}
& \Delta C_{2,6}=\Delta C_{1,5}=\Delta C_{3,7} \neq \Delta C_{4,8}, \\
& \Delta C_{2,4}=\Delta C_{1,3}=\Delta C_{5,7} \neq \Delta C_{6,8}, \\
& \Delta C_{3,4}=\Delta C_{1,2}=\Delta C_{5,6} \neq \Delta C_{7,8},
\end{aligned}
$$

то потоки вещества описываются формулами

$$
\begin{gathered}
q_{x}(x, y, z)=\frac{D}{a} \Delta C_{1,5}-\frac{D}{a} \frac{z}{c} \frac{y}{b}\left(\Delta C_{3,7}-\Delta C_{4,8}\right), \\
q_{y}(x, y, z)=\frac{D}{b} \Delta C_{1,3}-\frac{D}{b} \frac{z}{c} \frac{x}{a}\left(\Delta C_{5,7}-\Delta C_{6,8}\right), \\
q_{z}(x, y, z)=\frac{D}{c} \Delta C_{1,2}-D \frac{c}{\pi} Q \cos \pi \frac{z}{c}-\frac{D}{c} \frac{y}{b} \frac{x}{a}\left(\Delta C_{5,6}-\Delta C_{7,8}\right) .
\end{gathered}
$$


Если же в (27)-(29) только два значения из четырех $\Delta C_{i, j}, i=1 \ldots 7, j=2 \ldots 8$ равны друг другу, например

$$
\begin{aligned}
& \Delta C_{2,6}=\Delta C_{1,5} \neq \Delta C_{3,7} \neq \Delta C_{4,8}, \\
& \Delta C_{2,4}=\Delta C_{1,3} \neq \Delta C_{5,7} \neq \Delta C_{6,8}, \\
& \Delta C_{3,4}=\Delta C_{1,2} \neq \Delta C_{5,6} \neq \Delta C_{7,8},
\end{aligned}
$$

то потоки вещества определяются по формулам

$$
\begin{aligned}
& q_{x}(x, y, z)=\frac{D}{a}\left(\Delta C_{1,5}-\frac{y}{b}\left(\Delta C_{1,5}-\Delta C_{3,7}\right)-\frac{z}{c} \frac{y}{b}\left(\Delta C_{3,7}-\Delta C_{4,8}\right)\right) \\
& q_{y}(x, y, z)=\frac{D}{b}\left(\Delta C_{1,3}-\frac{x}{a}\left(\Delta C_{1,3}-\Delta C_{5,7}\right)-\frac{z}{c} \frac{x}{a}\left(\Delta C_{5,7}-\Delta C_{6,8}\right)\right) \\
& q_{z}(x, y, z)=\frac{D}{c}\left(\Delta C_{1,2}-\frac{x}{a}\left(\Delta C_{1,2}-\Delta C_{5,6}\right)-\frac{y}{b} \frac{x}{a}\left(\Delta C_{5,6}-\Delta C_{7,8}\right)\right)- \\
& -D \frac{c}{\pi} Q \cos \pi \frac{z}{c}
\end{aligned}
$$

При всех различных значениях $\Delta C_{i, j}, i=1 \ldots 7, j=2 \ldots 8$ в (27)-(29) потоки $q_{x}(x, y, z), q_{y}(x, y, z)$ и $q_{z}(x, y, z)$ будут вычисляться по формулам (24)-(26).

Покажем вид распределений диффузионных потоков вещества, соответствующие случаям, когда в равенствах (27) и (28) будут одинаковы по два значения $\Delta C_{i, j}$ из четырех, а в $(29)$ - три значения $\Delta C_{i, j}$ из четырех. Подобная ситуация выполнима, например, при данных

$$
\begin{gathered}
C_{1}=1, \quad C_{2}=2, \quad C_{3}=3, \quad C_{4}=4, \quad C_{5}=6, \quad C_{6}=7, \quad C_{7}=14, \quad C_{8}=11, \\
Q=5, \quad a=1 \mathrm{M}, \quad b=2 \mathrm{M}, \quad c=3 \mathrm{M}, \quad D=4 \cdot 10^{-6} \mathrm{~m}^{2} / c .
\end{gathered}
$$

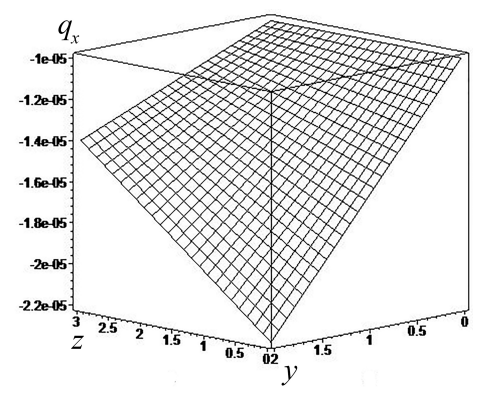

a)

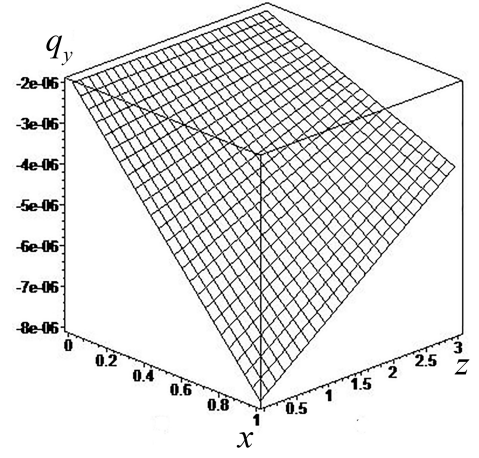

б)

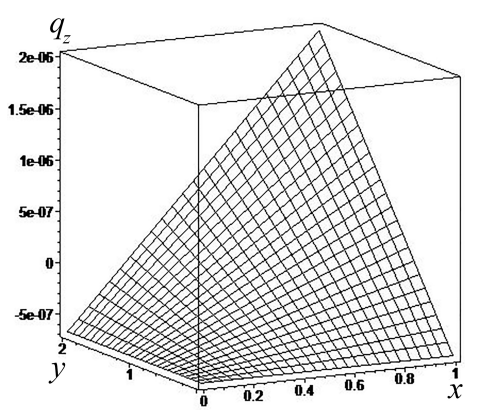

B)

Рис. 2. Потоки вещества в параллелепипеде: а) $q_{x}(x, y, z)$, б) $q_{y}(x, y, z)$, в) $q_{z}(x, y, z)$ в сечении $z=c / 2$

Для значений (40) на рис. 2а изображено распределение $q_{x}(x, y, z)$ для которого выполняются условия (36). На рис. $2 б$ представлен профиль $q_{y}(x, y, z)$, соответствующий условиям (37), а на рис. 2в показан график $q_{z}(x, y, z)$ в сечении $z=c / 2$, построенный при выполнении условий (34).

Распределение $C(x, y, z)$, рассчитанное по формуле (21), для случая (40) в сечениях плоскостями $x=a / 2, y=b / 2$ и $z=c / 2$ показано на рис. 3а, рис. 3б и рис. Зв соответственно. 


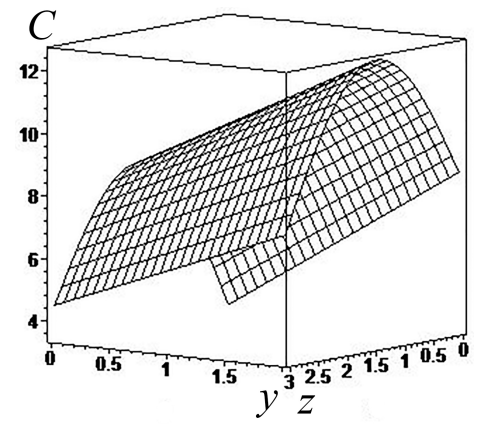

a)

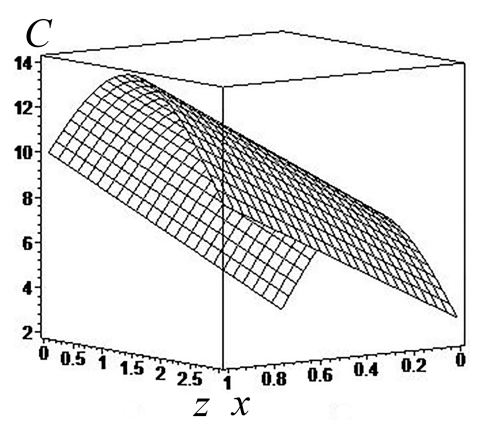

б)

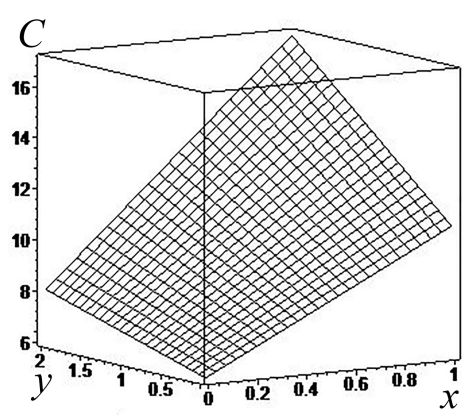

B)

Рис. 3. Концентрация вещества $C(x, y, z)$ в параллелепипеде в сечениях: а) $x=a / 2$, б) $y=b / 2$, в) $z=c / 2$

Из рис. 3 видно, что данные распределения концентрации плоскостей симметрии не имеют. Если же в (21) принять все начальные концентрации одинаковыми $C_{1}=C_{2}=$ $C_{3}=C_{4}=C_{5}=C_{6}=C_{7}=C_{8}$, то в этом случае получим распределения, имеющие плоскости симметрии, проходящие через центр параллелепипеда. Подобная ситуация соответствует выполнению условий (30) для диффузионных потоков вещества.

Заключение. Подбор численных значений коэффициентов функций, входящих в граничные условия и источник $F(x, y, z)$, следует вести с учетом равенств (11)-(13), (15).

Показано, что для внутреннего источника переменного только по оси $O Z$ потоки $q_{x}(x, y, z)$ и $q_{y}(x, y, z)$ не зависят от его амплитуды, а зависят только от концентраций вещества в вершинах параллелепипеда. На поток $q_{z}(x, y, z)$ влияют и концентрации вещества в вершинах, и амплитуда внутреннего источника. Для описания диффузионных потоков получены формулы, в которых выделены слагаемые, представляющие разности концентраций вещества в вершинах на соответствующих ребрах. Тем самым, было показано, что в описании потока $q_{x}(x, y, z)$ присутствуют разности концентраций в вершинах на ребрах коллинеарных только оси $O X$. Для описания $q_{y}(x, y, z)$ учитывается разности концентраций в вершинах на ребрах коллинеарных только оси $O Y$, а в записи $q_{z}(x, y, z)$ присутствуют разности концентраций вещества в вершинах на ребрах коллинеарных только оси $O Z$. Описание потоков $q_{x}(x, y, z), q_{y}(x, y, z)$, $q_{z}(x, y, z)$ полученными формулами, показывает, что возможны случаи качественно различных их профилей в зависимости от количества одинаковых значений разности концентраций в вершинах на соответствующих ребрах.

Получено, что концентрация вещества в центре параллелепипеда равна сумме среднеарифметического значения концентраций вещества в его вершинах и амплитуды $Q$ внутреннего источника умноженного на величину $c^{2} / \pi^{2}$.

\section{ЛИТЕРАТУРА}

[1] Губарев С. В., Берг Д. Б., Добряк П. В. Математическая модель и численный метод для решения задач диффузии и теплопроводности // Современные проблемы науки и образования. 2013. № 6. C. $176-186$. 
[2] Жуков В. Т., Новикова Н. Д., Феодоритова О. Б. Параллельный многосеточный метод для разностных эллиптических уравнений. Анизотропная диффузия // Препринты ИПМ им. М.В. Келдыша. 2012. № 76. С. 1-36.

[3] Юлдашев О. И., Юлдашева М. Б. Граничный метод взвешенных невязок с разрывными базисными функциями для высокоточного решения линейных краевых задач с уравнениями Лапласа и Пуассона // Вестник РУДН. Серия Математика. Информатика. Физика. 2013. № 4. С. 143-153.

[4] Алгазин О. Д., Копаев А. В. Решение задачи Дирихле для уравнения Пуассона в многомерном бесконечном слое // Математика и Математическое моделирование. МГТУ им. Н.Э. Баумана. 2015. № 4. C. 41-53.

[5] Земсков А. В., Файкин Г. М. Изгиб упругодиффузионной консольно-закрепленной балки Бернулли-Эйлера с учетом конечной скорости распространения диффузионных потоков // Вестник Чувашского государственного педагогического университета им. И.Я. Яковлева. Серия: Механика предельного состояния. 2020. № 4(46). С. 107-114.

[6] Исаев В. И., Шапеев В. П., Идимешев С. В. Варианты метода коллокаций и наименьших квадратов повышенной точности для численного решения уравнения Пуассона // Вычислительные технологии. 2011. Т. 16, № 1. С. 85-93.

[7] Zhong H., He Y. Solution of Poisson and Laplace equations by quadrilateral quadrature element // International Journal of Solids and Structures. 1998. Vol. 35, no. 21. P. 2805-2819.

[8] Ghasemi M. Spline-based DQM for multi-dimensional PDEs: Application to biharmonic and Poisson equations in 2D and 3D // Computers \& Mathematics with Applications. 2017. Vol. 73, no. 7. P. 15761592.

[9] Shi Z., Cao Y. Y., Chen Q. J. Solving 2D and 3D Poisson equations and biharmonic equations by the Haar wavelet method // Applied Mathematical Modelling. 2012. Vol. 36, no. 11. P. 5143-5161.

[10] Чернышов А. Д. Метод быстрых разложений для решения нелинейных дифференциальных уравнений // Журнал вычислительной математики и математической физики. 2014. Т. 54, № 1. C. $13-24$.

[11] Чернышов А. Д., Марченко А. Н., Горяйнов В. В. Температурный режим при естественной конвекции термовязкой несжимаемой жидкости в емкости прямоугольной формы // Тепловые процессы в технике. 2012. Т. 4, № 11. С. 482-486.

[12] Горяйнов В. В., Попов М. И., Чернышов А. Д. Решение задачи о напряжениях в остром клиновидном режущем инструменте методом быстрых разложений и проблема согласования граничных условий // Известия Российской академии наук. Механика твердого тела. 2019. № 5. С. 113-130.

[13] Шашкин А. И., Переяславская И. И. Применение метода быстрых разложений для нахождения напряженно-деформированного состояния упругого цилиндра с учетом силы тяжести // Вестник Чувашского государственного педагогического университета им. И.Я. Яковлева. Серия: Механика предельного состояния. 2014. № 4(22). С. 68-79.

[14] Применение быстрых разложений для построения точных решений задачи о прогибе прямоугольной мембраны под действием переменной нагрузки / А. Д. Чернышов, В. В. Горяйнов, С. Ф. Кузнецов [и др.] // Вестник томского государственного университета. Математика и механика. 2021. № 70. С. 127-142.

[15] Франк-Каменецкий Д. А. Диффузия и теплопередача в химической кинетике. Наука: Москва, 1987. $502 \mathrm{c}$. 
A. D. Chernyshov, V. V.Goryainov, S. F. Kuznetsov, O. Yu. Nikiforova

\title{
THREE-DIMENSIONAL EXACT SOLUTIONS OF THE DIFFUSION EQUATION
}

\author{
Voronezh State University of Engineering Technology, Voronezh, Russia \\ Voronezh State Technical University, Voronezh, Russia
}

\begin{abstract}
The authors solve the problem of diffusion in a parallelepiped-shaped body with boundary conditions of the 1st kind and an internal source of substance, depending on the parallelepiped points coordinates with the fast expansions method. The proposed exact solution in general form contains free parameters, which can be used to obtain many new exact solutions with different properties. An example of constructing an exact solution with a variable internal source depending on one coordinate $\mathrm{z}$ is shown in the work. An analysis of the features of diffusion flows in a parallelepiped with the indicated internal source is given. It was found that the concentration of a substance in the center of a parallelepiped is equal to the sum of the arithmetic mean of the concentration of a substance at its vertices and the amplitude of the internal source multiplied by the value $c^{2} / \pi^{2}$.
\end{abstract}

Keywords: analytical solution, exact solution, diffusion flows, variable internal source, parallelepiped, fast expansions

\section{REFERENCES}

[1] Gubarev S. V., Berg D. B., Dobryak P. V. Mathematical model and numerical methodfor solving problems of diffusion and heat conduction // Modern problems and science. 2013. no. 6. P. $176-186$.

[2] Zhukov V. T., Novikova N. D., Feodoritova O. B. Parallel multigridmethod for elliptic difference equations. Anisotropic diffusion // Keldysh Institute preprints M.V. Keldysh. 2012. no. 76. P. 1-36.

[3] Yuldashev O. I., Yuldasheva M. B. Boundary method of weighted residuals with discontinuous basis functions for high-precision solution of linear boundary value problems with the Laplace and Poisson equations // Bulletin of RUDN university. Series Mathematics. Informatics. Physics. 2013. no. 4. P. $143-153$.

[4] Algazin O. D., Kopaev A. V. Solution of the Dirichlet problem for the Poisson equationin a multidimensional infinite layer // Mathematics and Mathematical Modeling. MSTUnamed after N.E. Bauman. Electron journal. 2015. no. 4. P. 41-53.

[5] Zemskov A. V., Faykin G. M. Euler-Bernoulli cantilever beam bending considering theinner diffusion flows finite propagation speed // Bulletin of the Yakovlev Chuvash State Pedagogical University. Series: Mechanics of Limit State. 2020. no. 4(46). P. 107-114.

Chernyshov Alexander Danilovich

e-mail: chernyshovad@mail.ru, Dr. Sci. Phys. \& Math., Professor, Voronezh State University of Engineering Technology, Voronezh.

Goryainov Vitaly Valerievich

e-mail: gorvit77@mail.ru, Ph.D. Phys. \& Math., Assoc. Professor, Voronezh State Technical University, Voronezh.

Kuznetsov Sergey Fedorovich

e-mail: sfs134@mail.ru, Ph.D. Phys. \& Math., Assoc. Professor, Voronezh State University of Engineering Technology, Voronezh.

Nikiforova Olga Yurievna

e-mail: niki22@mail.ru, Senior Lecturer, Voronezh State University of Engineering Technology, Voronezh. 
[6] Isayev V. I., Shapeyev V. P., Idimeshev S. V. Variants of the method of collocationsand least squares of increased accuracy for the numerical solution of the Poisson equation // Computational technologies. 2011. Vol. 16, no. 1. P. 85-93.

[7] Zhong H., He Y. Solution of Poisson and Laplace equations by quadrilateral quadrature element // International Journal of Solids and Structures. 1998. Vol. 35, no. 21. P. 2805-2819.

[8] Ghasemi M. Spline-based DQM for multi-dimensional PDEs: Application to biharmonic and Poisson equations in 2D and 3D // Computers \& Mathematics with Applications. 2017. Vol. 73, no. 7. P. 15761592.

[9] Shi Z., Cao Y. Y., Chen Q. J. Solving 2D and 3D Poisson equations and biharmonic equations by the Haar wavelet method // Applied Mathematical Modelling. 2012. Vol. 36, no. 11. P. 5143-5161.

[10] Chernyshov A. Method of fast expansions for solving nonlinear differential equations // Computational Mathematics and Mathematical Physics. 2014. Vol. 54, no. 1. P. 13-24.

[11] Chernyshov A. D., Marchenko A. N., Goryainov V. V. Temperature regime undernatural convection of thermoviscous incompressible fluid in a rectangular containe // Thermal processes in technolog. 2012. Vol. 4, no. 11. P. 482-486.

[12] Goryainov V. V., Popov M. I., Chernyshov D. Solving the stress problem in a sharpwedgewhaped cutting tool using the quick decomposition method and the problem ofmatching boundary conditions // Mechanics of Solids. 2019. no. 5. P. 113-130.

[13] Shashkin A. I., Pereyaslavskaya I. I. Application of the method of fast decompositionsfor finding the stress-strain state of an elastic cylinder under the action of force of gravity // Bulletin of the Yakovlev Chuvash State Pedagogical University. Series: Mechanics of Limit State. 2014. no. 4(22). P. 68-79.

[14] Application offast expansions to obtain exact solutions to a problem on rectangular membrane deflectionunder alternating load / A. D. Chernyshov, V. V. Goryainov, S. F. Kuznetsov et al. // Tomsk State University Journal of Mathematics and Mechanics. 2021. no. 70. P. 127-142.

[15] Frank-Kamenetskii D. A. Diffusion and heat exchange in chemical kinetics. Science: Moscow, 1987. $502 \mathrm{p}$. 